4. Dimopoulos MA, Goldschmidt H, Niesvizky R, et al. Carfilzomib or bortezomib in relapsed or refractory multiple myeloma (ENDEAVOR): an interim overall survival analysis of an open-label, randomised, phase 3 trial. Lancet Oncol. 2017;18(10):1327-37.

5. National Institute for Health for Care Excellence. Carfilzomib for previously treated multiple myeloma. June 2017. Available at: https://www.nice. org.uk/guidance/ta457/documents/final-appraisal-determination-document. Accessed June 6, 2018.

6. Jakubowiak AJ, Houisse I, Májer I, et al. Cost-effectiveness of carfilzomib plus dexamethasone compared with bortezomib plus dexamethasone for patients with relapsed or refractory multiple myeloma in the United States. Expert Rev Hematol. 2017;10(12):1107-19.

7. Jakubowiak AJ, Campioni M, Benedict Á, et al. Cost-effectiveness of adding carfilzomib to lenalidomide and dexamethasone in relapsed multiple myeloma from a U.S. perspective. J Med Econ. 2016;19(11):1061-74.

\section{The Authors Respond}

We appreciate the opportunity to respond to the letter by Ailawadhi et al. regarding our article "Cost-effectiveness of Drugs to Treat Relapsed/Refractory Multiple Myeloma in the United States" and to provide clarifications where necessary to the points raised in their letter.

We do acknowledge 4 minor errors in our publication and have submitted appropriate corrections to JMCP. We will comment on 2 of those errors here, since the third and fourth were merely typos. The first relates to the progression-free survival (PFS) hazard ratio estimate for the panobinostat/ bortezomib/dexamethasone combination (PAN + BOR + DEX) versus lenalidomide (LEN) + DEX in the third-line treatment setting. Our study reported this estimate to be 0.54 (credible range $=0.50-0.83$ ) whereas the correct numbers should be 0.59 (credible range $=0.31-1.10$ ). ${ }^{1}$ This change also results in a slight revision to the comparative $\mathrm{PAN}+\mathrm{BOR}+\mathrm{DEX}$ versus LEN + DEX estimates, with the new estimates for total incremental costs and quality-adjusted life-years being $-\$ 76,328$ and 1.19 , respectively. Thus, PAN $+\mathrm{BOR}+\mathrm{DEX}$ is still found to be dominant versus LEN + DEX in this comparison.

The second correction is to add the word "treatment" before the word "cost" in the "Discussion" section paragraph in which we discuss the Jakubowiak et al. study. ${ }^{2}$ The new sentence should read as follows:

"Finally, we note that 1 of the findings of the Jakubowiak et al. analysis appears to be counterintuitive, in that CFZ + LEN + DEX patients spend approximately 4 years in the postprogression state in the model versus approximately 3 years for LEN + DEX; however, the postprogression treatment costs for LEN+DEX are reported to be higher."

The overall postprogression costs are slightly higher for carfilzomib (CFZ) + LEN + DEX by $\$ 856$; however, the key point of the sentence remains valid, especially given the high cost of treating relapsed/refractory multiple myeloma; that is, patients receiving CFZ+LEN + DEX spent about 10 months more in the progression health state but had lower treatment costs and only slightly higher overall health state costs. This increase appears to be due to the use of different distributions of subsequent treatments by treatment arm and the assumption that subsequent treatments would stop after 17 cycles. Jakubowiak et al. suggest that they ran a scenario analysis using the same distribution, but the results of this scenario analysis are not provided.

We take this opportunity to point out an additional issue with the Jakubowiak et al. analysis. Their base case analysis uses 2 different health state utility values for the postprogression health state, even though patients in this health state should be relatively homogenous with respect to their healthrelated quality of life while in the same health state-that is, the patients will have already progressed on CFZ + LEN + DEX and LEN +DEX and would now be assumed to be treated similarly. Notably, the values applied to the CFZ + LEN + DEX intervention group after progression were higher than those for LEN + DEX (0.664 vs. 0.643). We note this additional bias in response to Ailawadhi et al.'s suggestion that "the cost-effectiveness of CFZ +DEX versus BOR +DEX has been unequivocally established," with reference to the Jakubowiak et al. study. ${ }^{2}$

Ailawadhi et al. also suggested additional limitations to the network meta-analysis (NMA) used in our study in terms of comparability. We stated in our article that "trial populations were similar with respect to age, ECOG performance status, ISS stage, receipt of previous stem cell transplant, and number and distribution of previous regimens." We acknowledged that definitions of disease risk varied. Other studies have used standard and comparable methods to assess PFS. Because PFS was unavailable for MM-009 and MM-010, time-to-progression was used; this approach has been used in other indirect comparisons of the agents of interest, including in the NICE panobinostat review cited by Ailawakhi et al. in their letter. Other recently published NMAs of relapsed/refractory multiple myeloma have taken a similar approach and have included a similar study set in their networks. ${ }^{3-6}$

In response to the comment that our results lack face validity because "LEN + DEX is estimated to be less effective than $\mathrm{BOR}+\mathrm{DEX}$ even though all listed trials show that LEN + DEX achieved double the PFS than BOR+DEX," we note that $\mathrm{LEN}+\mathrm{DEX}$ is not estimated to be less effective than BOR + DEX. The NMA did not show the 2 regimens to be statistically different. Importantly, trials of LEN +DEX have had follow-up durations that were 2-3 times longer than the BOR+DEX trials and were treat-to-progression, whereas BOR+DEX was given for a fixed number of cycles. Furthermore, and as we have already mentioned, we have corrected the PFS hazard ratio for $\mathrm{PAN}+\mathrm{BOR}+\mathrm{DEX}$ versus $\mathrm{LEN}+\mathrm{DEX}$, which now shows that the credible range includes 1 . We also note that our study has substantial cautionary language about this result, as shown in this example from the article: 
"Results for PAN+BOR+DEX in the third-line setting should be interpreted with great caution because of censoring issues and high rates of toxicity-related discontinuation in the overall and third-line subgroup population of the PANORAMA-1 study. $\mathrm{PAN}+\mathrm{BOR}+\mathrm{DEX}$ is also only 1 of 2 regimens without direct comparative evidence versus LEN + DEX; therefore, greater reliance on the study network and its assumptions regarding minimal heterogeneity across study populations and constant hazards over time was required. While censoring was factored into our analytic approach, the relative treatment effect of $\mathrm{PAN}+\mathrm{BOR}+\mathrm{DEX}$ versus LEN + DEX had much greater uncertainty than the other comparisons."

Regarding the comment that "carfilzomib+DEX twice weekly was not even considered for the analysis," we received clinical expert input that a triplet is preferable to a doublet when a patient can tolerate it, so CFZ + LEN + DEX was therefore much more likely to be used in the settings of interest than CFZ+DEX. Hence, we focused on comparing new triplet therapies to the 2 standard of care approaches-LEN+DEX and $\mathrm{BOR}+\mathrm{DEX}$.

Finally, we note that the treatment costs for CFZ were capped at 18 months, in line with the suggested dosing.

Overall, we feel that our analysis and the conclusions we have drawn are robust and well founded.

\section{Josh J. Carlson, MPH, PhD}

The Comparative Health Outcomes, Policy, and Economics (CHOICE) Institute, University of Washington, Seattle Carlsojj@u.washington.edu

\section{Gregory F. Guzauskas, MSPH, PhD}

The Comparative Health Outcomes, Policy, and Economics (CHOICE) Institute, University of Washington, Seattle

Richard H. Chapman, PhD, MS

Institute for Clinical and Economic Review Boston, Massachusetts

\section{Patricia G. Synnott, MS, MALD}

Institute for Clinical and Economic Review Boston, Massachusetts

\section{Shanshan Liu, MS \\ Institute for Clinical and Economic Review Boston, Massachusetts}

Elizabeth T. Russo, MD

Institute for Clinical and Economic Review Boston, Massachusetts

\section{Steven D. Pearson, MD, MSc}

Institute for Clinical and Economic Review

Boston, Massachusetts

\section{Elizabeth D. Brouwer, MPH}

The Comparative Health Outcomes, Policy, and Economics

(CHOICE) Institute, University of Washington, Seattle

Daniel A. Ollendorf, PhD

Institute for Clinical and Economic Review

Boston, Massachusetts

\section{DISCLOSURES}

Funding for the Carlson et al. study was provided in part by the Institute for Clinical and Economic Review. Ollendorf, Synnott, Chapman, and Pearson disclosed grants from Blue Shield of California Foundation, California Health Care Foundation, Laura and John Arnold Foundation, Aetna, AHIP, Anthem, Blue Shield of California, CVS Caremark, Express Scripts, Harvard Pilgrim Health Care, OmedaRx, United Healthcare, Kaiser Permanente, Premera, AstraZeneca, Genentech, GlaxoSmithKline, Johnson \& Johnson, Merck, National Pharmaceutical Council, Takeda, Pfizer, Novartis, Lilly, Spark Therapeutics, Sanofi, Prime Therapeutics, and Health Care Service Corporation. Carlson disclosed grants from the Institute for Clinical and Economic Review and personal fees from Seattle Genetics, Genentech, and Pfizer. Russo, Guzauskas, Liu, and Brouwer have nothing to disclose.

\section{REFERENCES}

1. Carlson JJ, Guzauskas GF, Chapman RH, et al. Cost-effectiveness of drugs to treat relapsed/refractory multiple myeloma in the United States. J Manag Care Spec Pharm. 2018;24(1):29-38. Available at: https://www.jmcp.org/ doi/10.18553/jmcp.2018.24.1.29

2. Jakubowiak AJ, Houisse I, Majer I, et al. Cost-effectiveness of carfilzomib plus dexamethasone compared with bortezomib plus dexamethasone for patients with relapsed or refractory multiple myeloma in the United States. Expert Rev Hematol. 2017;10(12):1107-19.

3. Botta C, Ciliberto D, Rossi M, et al. Network meta-analysis of randomized trials in multiple myeloma: efficacy and safety in relapsed/refractory patients. Blood Adv. 2017;1(7):455-66.

4. Dimopoulos MA, Kaufman JL, White D, et al. A comparison of the efficacy of immunomodulatory-containing regimens in relapsed/refractory multiple myeloma: a network meta-analysis. Clin Lymphoma Myeloma Leuk. 2018;18(3):163-73.el66.

5. van Beurden-Tan CHY, Franken MG, Blommestein HM, Uyl-de Groot CA, Sonneveld P. Systematic literature review and network meta-analysis of treatment outcomes in relapsed and/or refractory multiple myeloma. J Clin Oncol. 2017;35(12):1312-19.

6. Ruggeri K, Maguire A, Schmitz S, et al. Estimating the relative effectiveness of treatments in relapsed/refractory multiple myeloma through a systematic review and network meta-analysis. Blood. 2015;126(23):2103 [abstract]. Available at: http://www.bloodjournal.org/ content/126/23/2103?sso-checked=true. Accessed June 8, 2018. 